\title{
Molecular Imaging in Cancer Drug Development
}

\author{
Stijn J.H. Waaijer ${ }^{* 1}$, Iris C. Kok ${ }^{* 1}$, Bertha Eisses ${ }^{1}$, Carolina P. Schröder ${ }^{1}$, Mathilde Jalving ${ }^{1}$, Adrienne H. Brouwers ${ }^{2}$, \\ Marjolijn N. Lub-de Hooge ${ }^{2,3}$, and Elisabeth G.E. de Vries ${ }^{1}$ \\ ${ }^{I}$ Department of Medical Oncology, University Medical Center Groningen, University of Groningen, Groningen, The Netherlands; \\ ${ }^{2}$ Department of Nuclear Medicine and Molecular Imaging, University Medical Center Groningen, University of Groningen, \\ Groningen, The Netherlands; and ${ }^{3}$ Department of Clinical Pharmacy and Pharmacology, University Medical Center Groningen, \\ University of Groningen, Groningen, The Netherlands
}

\begin{abstract}
Development of new oncology drugs has increased since the improved understanding of cancer's complex biology. The oncology field has become the top therapeutic research area for new drugs. However, only a limited number of drugs entering clinical trials will be approved for use as the standard of care for cancer patients. Molecular imaging is increasingly perceived as a tool to support go/ no-go decisions early during drug development. It encompasses a wide range of techniques that include radiolabeling a compound of interest followed by visualization with SPECT or PET. Radiolabeling can be performed using a variety of radionuclides, which are preferably matched to the compound on the basis of size and half-life. Imaging can provide information on drug behavior in vivo, wholebody drug target visualization, and heterogeneity in drug target expression. This review focuses on current applications of molecular imaging in the development of small molecules, antibodies, and antihormonal anticancer drugs.
\end{abstract}

Key Words: cancer; drug development; molecular imaging; PET

J Nucl Med 2018; 59:726-732

DOI: 10.2967/jnumed.116.188045

I oncology there is by far the highest number of new drugs in development trajectories (1). These include more than 800 medicines and vaccines for cancer, with over 240 immunooncology drugs $(2,3)$. Illustratively, in 2016, 20 drugs targeting programmed death 1 or programmed death ligand 1 were being studied in 803 registered clinical trials with slots for 166,736 patients (4).

Unfortunately, numerous drugs fail to get clinical approval. Only around $12 \%$ of the compounds entering the clinical trial phase make it to regulatory submission (5). These often-late failures have made drug discovery extremely expensive. Sums of over $\$ 2$ billion per single drug approval have been named (5), although recent estimations suggest a median cost of about $\$ 648.0$ million, with a median revenue after approval of $\$ 1,658.4$ million (6). Typically, a new drug is explored in 3 phases: namely, phase 1 to determine a safe dose and side effects, phase 2 to measure antitumor

Received Nov. 6, 2017; revision accepted Jan. 16, 2018.

For correspondence or reprints contact: Elisabeth G.E. de Vries, Department of Medical Oncology, University of Groningen, University Medical Center Groningen, P.O. Box 30.001, 9700 RB Groningen, The Netherlands. E-mail: e.g.e.de.vries@umcg.nl

${ }^{*}$ Contributed equally to this work.

Published online Jan. 25, 2018.

COPYRIGHT (C 2018 by the Society of Nuclear Medicine and Molecular Imaging. effect, and a randomized phase 3 study to define its effect compared with standard treatment.

To improve and streamline cancer drug development, the " 3 pillars of survival" (7), and more recently the pharmacological audit trail, were proposed (8). This trail consists of a set of key questions to be asked during discovery and development, covering aspects such as population identification, pharmacokinetics, pharmacodynamics, and combination therapy (8). Molecular imaging can give additional information about, for example, target validation; tumor targeting; whole-body target expression, including the currently more appreciated heterogeneity; whole-body drug distribution; pharmacokinetic features such as central nervous system (CNS) penetration; and pharmacodynamic effects (Fig. 1).

Initially, generic PET tracers such as ${ }^{18} \mathrm{~F}$-FDG for glucose metabolism and ${ }^{18}$ F-labeled $3^{\prime}$-deoxy-3' fluorothymidine for proliferation were used in cancer drug development. After a general introduction on imaging modalities, this review addresses more specific tracers, with emphasis on radiolabeled drugs for smallmolecule drugs, monoclonal antibodies (mAbs), and antihormonal anticancer drugs.

\section{SEARCH STRATEGY}

We searched the English literature in PubMed, the Dutch trial registry, and the EudraCT and ClinicalTrials.gov databases. The abstracts of annual meetings from 2015 until the present of the American Society of Clinical Oncology, American Association of Cancer Research, European Society of Medical Oncology, and San Antonio Breast Cancer Symposium were additionally screened. The search strategy focused on molecular imaging in the context of target expression, pharmacokinetics, and pharmacodynamics in cancer. Reference lists of articles and citing articles were manually searched for relevance.

\section{GENERAL INTRODUCTION TO IMAGING MODALITIES}

Different molecular imaging modalities can be used to support drug development. These include SPECT, PET, MRI, and optical imaging using fluorescence or bioluminescence. SPECT and PET are the most commonly used techniques, of which PET provides better resolution and quantification. For SPECT and PET imaging, different radionuclides are used. Smaller compounds and peptides tend to be labeled with ${ }^{99 \mathrm{~m}} \mathrm{Tc}$ for SPECT and ${ }^{11} \mathrm{C}$, ${ }^{68} \mathrm{Ga}$, or ${ }^{18} \mathrm{~F}$ for PET, with half-lives of 360, 20, 68, and $110 \mathrm{~min}$, respectively. Larger molecules such as mAbs can be efficiently labeled with a wider range of SPECT and PET radionuclides. These include ${ }^{123} \mathrm{I}$ and ${ }^{111} \mathrm{In}$ for SPECT and ${ }^{64} \mathrm{Cu},{ }^{124} \mathrm{I},{ }^{86} \mathrm{Y}$, and ${ }^{89} \mathrm{Zr}$ for PET. Considering the different half-lives of these radionuclides, ranging 


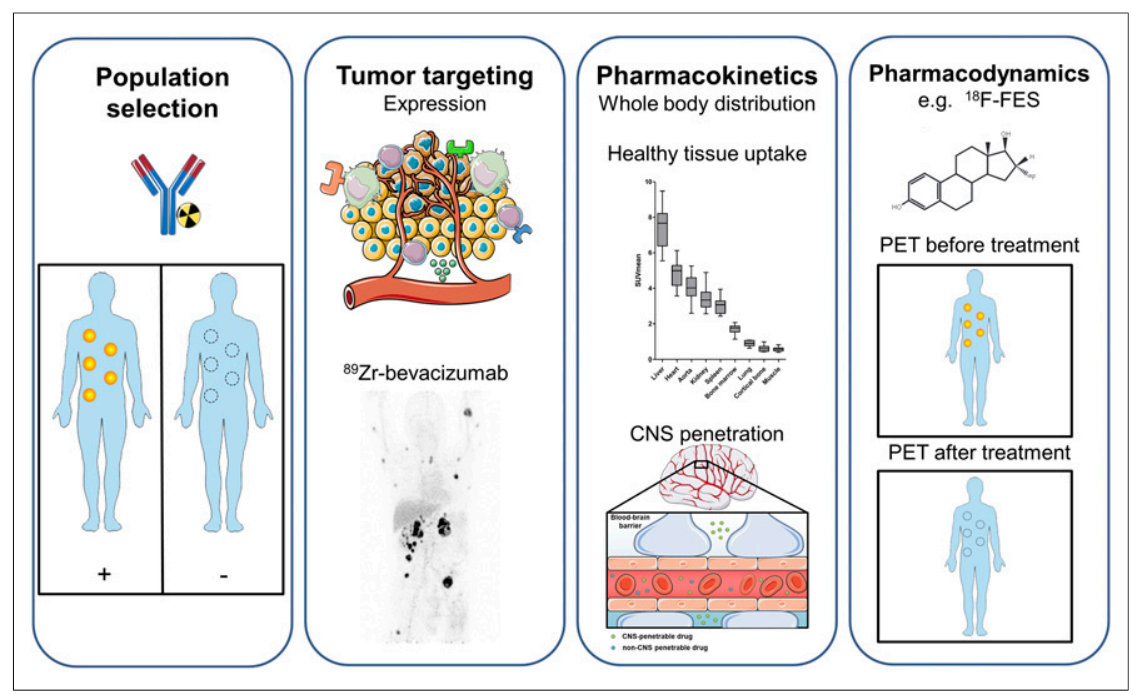

FIGURE 1. Information that can be extracted using molecular imaging, categorized by population selection, tumor targeting, pharmacokinetics, and pharmacodynamics $(7,8)$. (First panel) Molecular imaging with, for instance, radiolabeled antibodies can potentially identify responders and nonresponders. (Second panel) For tumor targeting, several tumor aspects can be visualized with molecular imaging, such as tumor cell receptors, environmental factors, and immune cells. Example is PET visualization of ${ }^{89} \mathrm{Zr}$-bevacizumab targeting vascular endothelial growth factor $\mathrm{A}$ in tumor microenvironment in patient with metastatic renal cell carcinoma (bottom; adapted from (60)). (Third panel) For pharmacokinetics, molecular imaging can provide information about wholebody distribution, normal-tissue accumulation of, for instance, ${ }^{89} \mathrm{Zr}$-bevacizumab (top; adapted from (60)), and penetration of CNS (bottom). Data on normal-tissue uptake might explain drug behavior. (Fourth panel) Pharmacodynamic information can be obtained by performing PET before and after treatment. Example is use of ${ }^{18} \mathrm{~F}-\mathrm{FES}$ for tumor uptake per lesion on antiestrogen therapy, resulting in less uptake. By this pharmacodynamic assessment, therapeutic dose with maximal decrease in tracer uptake can support further clinical studies. This figure was prepared using template on Servier medical art website (https://smart.servier.com/)

from $12.7 \mathrm{~h}$ for ${ }^{64} \mathrm{Cu}$ to $100.3 \mathrm{~h}$ for ${ }^{124} \mathrm{I}$, a radionuclide can be matched to the large molecule of interest on the basis of serum half-life. ${ }^{89} \mathrm{Zr}$ has a half-life of $78.4 \mathrm{~h}$, which matches the half-life of most full-sized mAbs and residualizes on internalization, making it an attractive radionuclide for $\mathrm{mAb}$ imaging. For fluorescent labeling, near-infrared fluorescent dyes such as IRDye800CW are of increasing interest and already clinically applied.

An interesting concept for clinical evaluation of tracers for first-in-human trials is microdosing (9). A microdose is defined as a molecule dose of less than $100 \mu \mathrm{g}$, or lower than $1 / 100 \mathrm{of}$ the pharmacologically active dose. For larger molecules such as proteins, a molar limit of $30 \mathrm{nmol}$ is applied. Given limited drug exposure, less extensive toxicity data are required. These so-called phase 0 trials with few participants can provide preliminary

\section{NOTEWORTHY}

- In oncology there is by far the highest number of new drugs in development trajectories.

- Molecular imaging can give information about whole-body drug target presence, whole-body drug distribution, pharmacokinetic features, and pharmacodynamic effects.

- For molecular imaging with SPECT and PET, radionuclides with different half-lives are available, allowing appropriate matching to serum half-life of the drug of interest to be labeled. information about drug pharmacokinetics and thus support drug development in early go/no-go decision making. Information from microdose studies can be translated to therapeutic doses only with dose-linear pharmacokinetics. As biologics often display non-linear pharmacokinetics, extrapolation from microdose to therapeutic dose is less reliable. Remarkably few studies using tracer microdosing in drug development have been published, but there may well be underreporting.

\section{MOLECULAR IMAGING USING SMALL-MOLECULE CANCER DRUGS}

Within the group of small molecules, kinase inhibitors form a large part. Currently, over 40 kinase inhibitors are approved for cancer treatment, with many more being in early clinical development. Radiolabeling of these small-molecule cancer drugs $(<1 \mathrm{kDa})$ with ${ }^{11} \mathrm{C}$ or ${ }^{18} \mathrm{~F}$ is challenging. Ideally, an isotopologue of the small-molecule cancer drug would be used. However, when this is not possible, analogs are used, which can have altered target affinity and pharmacokinetic characteristics. Despite the difficulties in labeling small-molecule cancer drugs, several approved drugs are radiolabeled, including ${ }^{11} \mathrm{C}$-imatinib, ${ }^{18} \mathrm{~F}$-sunitinib, ${ }^{11} \mathrm{C}$-sorafenib, ${ }^{11} \mathrm{C}$-erlotinib, ${ }^{11} \mathrm{C}$-lorlatinib, and ${ }^{11} \mathrm{C}$-vandetanib (10). However, clinical evaluation of these tracers is limited. We here highlight the application of radiolabeled drugs targeting epidermal growth factor receptor (EGFR), anaplastic lymphoma kinase (ALK), and poly(adenosine diphosphate ribose) polymerase (PARP).

\section{EGFR Inhibitors}

EGFR inhibitors are administered to patients with non-small cell lung cancer (NSCLC) bearing an activating EGFR mutation, which has a prevalence of approximately $10 \%-15 \%$. There are several approved EGFR inhibitors, including the first-generation inhibitors erlotinib and gefitinib, the second-generation inhibitor afatinib, and the third-generation inhibitor osimertinib. Osimertinib is approved in patients with the most commonly acquired T790M mutation, which is involved in resistance to first- and second-generation EGFR inhibitors.

Discordance can occur between the mutational status of the primary tumor and brain metastases measured by genomic analysis in biopsies (11). Examples include EGFR alterations in brain lesions that were absent in the primary tumor (11). Molecular imaging can potentially provide information on the mutational status of $E G F R$ lesions and thereby facilitate drug development by improving patient selection. ${ }^{18} \mathrm{~F}-\mathrm{N}$-(3-chloro-4-fluorophenyl)7-(2(2-(2-(2-(4-fluorine)ethoxy)ethoxy)-ethoxy)-6-(3-morpholinopropoxy)quinazolin-4-amine $\left({ }^{18} \mathrm{~F}\right.$-IRS) is a novel radiotracer developed to image the EGFR exon 19 deletion, an EGFR aberration leading to constitutive EGFR activation (12). Preclinically, ${ }^{18} \mathrm{~F}$ IRS showed preferential uptake in tumors with EGFR exon 19 deletion (12). Uptake with a mean $\mathrm{SUV}_{\max }$ of 2.4 was also 
observed in tumor lesions with an exon 19 deletion from 3 NSCLC patients (12). Imaging of the mutational status of NSCLC was also pursued using ${ }^{18} \mathrm{~F}-\mathrm{ODS} 2004436$, a compound chosen on the basis of EGFR selectivity. Preclinically, ${ }^{18}$ F-ODS2004436 showed increased uptake in rats with EGFR mutated lung cancer xenografts compared with $E G F R$ wild-type xenografts (13). Clinical evaluation of ${ }^{18} \mathrm{~F}-\mathrm{ODS} 2004436$ in NSCLC is ongoing. Whether molecular imaging can successfully assess whole-body EGFR mutational status and therefore aid in patient selection has to be studied more extensively.

Molecular imaging using radiolabeled EGFR tyrosine kinase inhibitors (TKIs) has been performed with ${ }^{11} \mathrm{C}$-erlotinib, ${ }^{11} \mathrm{C}$-gefitinib, ${ }^{18} \mathrm{~F}$-afatinib, the third-generation inhibitor ${ }^{11} \mathrm{C}$-osimertinib, ${ }^{11} \mathrm{C}$ AZD3759, and (development halted) ${ }^{11} \mathrm{C}$-rociletinib. Finally, ${ }^{11} \mathrm{C}$-labeled 4- $N$-(3-bromoanilino)-6,7-dimethoxyquinazoline $\left({ }^{11} \mathrm{C}\right.$ PD153035), a PET tracer based on a reversible EGFR TKI, was studied in patients with NSCLC receiving erlotinib treatment. Only ${ }^{11} \mathrm{C}$-erlotinib, ${ }^{18} \mathrm{~F}$-afatinib, and ${ }^{11} \mathrm{C}$-PD153035 have been studied in the clinical setting.

Most experience in patients with NSCLC is with ${ }^{11} \mathrm{C}$-erlotinib. In a study on 10 patients, full kinetic modeling of ${ }^{11} \mathrm{C}$-erlotinib via continuous arterial sampling demonstrated volume of distribution as the best parameter to represent ${ }^{11} \mathrm{C}$-erlotinib uptake (14). The study showed that the volume of distribution was higher in the 5 patients with an activating $E G F R$ mutation than in patients with $E G F R$ wild-type tumors. This effect was independent of EGFR expression as measured by immunohistochemistry or of perfusion as assessed by ${ }^{15} \mathrm{O}-\mathrm{H}_{2} \mathrm{O}$ PET. In a subsequent study, ${ }^{11} \mathrm{C}$-erlotinib was studied in 10 patients during erlotinib treatment (15). Erlotinib treatment decreased tumor tracer uptake in all patients, whereas perfusion measured with ${ }^{15} \mathrm{O}-\mathrm{H}_{2} \mathrm{O}$ remained similar. In another study, on 13 patients with NSCLC with unknown EGFR mutational status, baseline ${ }^{11} \mathrm{C}$-erlotinib uptake was visualized in the tumors of 4 patients (16). Of these 4 patients, 3 showed stable disease on erlotinib treatment. Using another radiolabeled EGFR TKI, ${ }^{11}$ C-PD-153035, higher tumor uptake on PET was associated with prolonged progression-free and overall survival after erlotinib treatment in a pilot study with 21 NSCLC patients (17). ${ }^{18} \mathrm{~F}$ afatinib is being studied in NSCLC patients in an ongoing trial (Dutch trial register identifier, NTR5203).

Brain penetration of the third-generation EGFR TKI osimertinib was studied using ${ }^{11} \mathrm{C}$-osimertinib in cynomolgus monkeys (18). CNS penetration of ${ }^{11} \mathrm{C}$-osimertinib was compared with that of ${ }^{11} \mathrm{C}$-rociletinib and ${ }^{11} \mathrm{C}$-gefitinib. At PET microdosing conditions with less than $3 \mu \mathrm{g}$ of ${ }^{11} \mathrm{C}$-osimertinib, higher brain exposure was seen than for the other EGFR TKIs. The increased osimertinib brain penetration also resulted in regression of brain lesions in a mouse brain metastasis model. In the same study, preliminary clinical efficacy for osimertinib was shown by noncomplete response-nonprogressive disease in the brain lesions of 2 patients with NSCLC (18). The prominent effects of osimertinib for NSCLC brain metastases have now been proven even in a large phase 3 trial as first-line treatment (19). Molecular imaging was also performed with another third-generation EGFR TKI, AZD3759, which was designed for improved CNS penetration. Clear healthy brain uptake in cynomolgus monkeys $(n=2)$ was shown by ${ }^{11} \mathrm{C}$-AZD3759 PET (20). In the phase 1 trial with 20 patients with NSCLC and CNS involvement, an impressive 63\% intracranial objective response rate with AZD3759 (12/19 evaluable patients) was observed, indicating sufficient brain penetration of AZD3759 (21).
All in all, EGFR-TKI PET demonstrates its value in pharmacokinetics, in particular CNS penetration. In addition, there is some evidence regarding preferred uptake for EGFR-mutated tumors and discrimination between responders and nonresponders. However, larger studies are needed.

\section{ALK Inhibitors}

Several ALK inhibitors have recently been approved by the Food and Drug Administration for the treatment of the 5\% of patients with NSCLC who have a genetic aberration involving $A L K$, such as echinoderm microtubule-associated proteinlike 4 (EMLA)-ALK translocation. This translocation can act as an oncogenic driver, thereby promoting cancer cell growth (22). After approval of the first-generation ALK inhibitor crizotinib, the second-generation ALK inhibitors ceritinib, brigatinib, and alectinib became available for patients resistant to crizotinib. However, patients can also acquire resistance to the second-generation ALK inhibitors. Therefore, the third-generation ALK inhibitor lorlatinib was developed, which shows activity against all known acquired $A L K$ mutations (23). The brain is a common metastatic site in NSCLC, and therefore activity against intracerebral lesions is critical for patient survival and quality of life. Alectinib was detected in cerebrospinal fluid (24) and improved patient outcome regarding CNS progression and progression-free survival (25). However, a new mutation will eventually arise on second-generation ALK inhibitor therapy, leading to third-generation ALK inhibitor lorlatinib treatment. Lorlatinib has been specifically developed for improved CNS penetration (26). To assess CNS penetration of lorlatinib noninvasively, ${ }^{11} \mathrm{C}$ and ${ }^{18} \mathrm{~F}$ isotopologues of lorlatinib were developed $(27) .{ }^{11} \mathrm{C}$ lorlatinib administered to nonhuman primates showed that $\mathrm{CNS}$ uptake of ${ }^{11} \mathrm{C}$-lorlatinib peaked at $10 \mathrm{~min}$ after injection, with the highest uptake being in the cerebellum (27). Tumor imaging in a human EML4-ALK-positive NSCLC xenograft mouse model showed that tumor uptake $(2.2 \%-2.4 \%$ injected dose per gram of tissue) could be blocked by adding unlabeled lorlatinib $(<0.4 \%$ injected dose per gram of tissue) (27). Besides ${ }^{11} \mathrm{C}$-lorlatinib, ${ }^{18} \mathrm{~F}$-lorlatinib was successfully synthesized, but it has not been studied yet in vivo.

\section{PARP Inhibitors}

Recently, PARP inhibitors have entered the clinic with Food and Drug Administration-approved drugs, including olaparib and niraparib. Using molecular imaging, whole-body PARP expression and pharmacodynamic changes on PARP treatment have been assessed in preclinical and clinical settings, as recently reviewed (28). An example includes ${ }^{18} \mathrm{~F}$-fluorthanatrace, which demonstrated specific tumor uptake by blocking tumor uptake by olaparib in preclinical breast cancer models (29). In patients with ovarian cancer, ${ }^{18} \mathrm{~F}$ fluorthanatrace lesion uptake corresponded to DNA damage as assessed in tissue histology by the DNA damage marker $\gamma$-H2AX (30). Although ${ }^{18} \mathrm{~F}$-fluorthanatrace has not been studied clinically in the context of PARP inhibition, this novel technology has potential to assess whole-body PARP expression and evaluate pharmacodynamic changes on PARP inhibition in patients who are eligible for PARP treatment. Particularly in the setting of breast cancer, in which the role of PARP inhibitors has not been firmly established, this ability could provide relevant insights. Imaging of PARP expression is being further explored in several ongoing clinical trials.

\section{MOLECULAR IMAGING WITH mAbS}

$\mathrm{mAbs}$ are directed against a specific target and in general have a long half-life of around $3 \mathrm{wk}$. They form a group of anticancer 
drugs that includes more than $24 \mathrm{mAbs}$ that are registered for standard care in curative and noncurative settings and around 200 more that are in clinical development (3). mAbs target antigens on the tumor cell affecting receptor signaling and turnover (e.g., trastuzumab), the vasculature or stroma (e.g., bevacizumab), or characteristics on other cells such as $\mathrm{T}$ cells. The immune checkpoint modulators have raised a lot of recent attention given their antitumor effects across numerous tumor types, and mAbs are increasingly being used to deliver a toxic payload in the form of a cytotoxic agent or radioisotope bound to a $\mathrm{mAb}$ forming an antibody-drug conjugate (ADC) or radioimmunotherapy, respectively.

\section{Growth Factor Receptors}

Sufficient target expression and efficacious dose ranges at the $\mathrm{mAb}$ site of action are a prerequisite for the drug to work. Moreover, given the fact that there are often few to no side effects, it is problematic to determine the optimal $\mathrm{mAb}$ dose to be administered to patients.

The radiolabeled mAb trastuzumab has been studied extensively. In treatment-naïve patients with human epidermal growth factor receptor 2 (HER2)-positive metastatic breast cancer, the optimal protein dose for ${ }^{89} \mathrm{Zr}$-trastuzumab PET was $50 \mathrm{mg}$ (31). In these patients, because of the dose-dependent pharmacokinetics of trastuzumab, with a known average terminal half-life of $1.1 \mathrm{~d}, 10 \mathrm{mg}$ of trastuzumab were excreted immediately, not allowing proper imaging. After multiple therapeutic doses of trastuzumab, its average terminal half-life increases to $28.5 \mathrm{~d}$ in a steady state, providing an excellent setting for imaging with $10 \mathrm{mg}$ of trastuzumab (32). From a SPECT study with serial ${ }^{111}$ In-trastuzumab SPECT imaging before and after $12 \mathrm{wk}$ of treatment with trastuzumab and paclitaxel, we learned that HER2 target saturation is limited (33).

In a study with ${ }^{89} \mathrm{Zr}$-lumretuzumab targeting human epidermal growth factor receptor 3, increasing doses of lumretuzumab did not lead to a plateau of tumor ${ }^{89} \mathrm{Zr}$-lumretuzumab uptake, possibly because of highly dynamic receptor expression, reflecting the difficulty in defining the maximum required $\mathrm{mAb}$ dose in the clinic (34).

Not only cell membrane targets but also targets in the tumor microenvironment can be visualized, as was shown in multiple studies performed with ${ }^{89} \mathrm{Zr}$-bevacizumab targeting vascular endothelial growth factor A. A pilot study with pretreatment ${ }^{89} \mathrm{Zr}$-bevacizumab PET in 7 NSCLC patients showed a high tumor-to-background ratio in primary tumor and metastases, suggesting specific tumor uptake (35). With repeated ${ }^{89} \mathrm{Zr}$-bevacizumab PET imaging of metastatic renal cell cancer before treatment and after 2 and $6 \mathrm{wk}$ of treatment, there was a decrease in target visualization highly suggestive of reduced access by inhibition of angiogenesis (36). Repeated ${ }^{89} \mathrm{Zr}$-bevacizumab PET imaging was also performed on 14 patients with advanced neuroendocrine tumors at baseline and during treatment with everolimus, and intra- and interpatient heterogeneity of ${ }^{89} \mathrm{Zr}$-bevacizumab lesion uptake was shown (37). Everolimus treatment is known to reduce vascular endothelial growth factor A secretion, and indeed, everolimus treatment for 12 wk reduced ${ }^{89} \mathrm{Zr}$-bevacizumab uptake compared with baseline, illustrating that ${ }^{89} \mathrm{Zr}$-bevacizumab tracer uptake functioned as a pharmacodynamic marker.

\section{Immunooncology}

In the rapidly evolving field of immunooncology there are still major questions, including which patients and tumor types benefit from immune checkpoint inhibitors. Because many studies with new cancer drugs are performed on mouse models with a mouse immune system, the gap between mouse and human has to be bridged. Use of humanized mice with a human immune system is a step forward in translating results to predict drug behavior in humans more reliably; however, this model lacks the presence of human cytokines, human leukocyte antigen proteins, and human organs.

Checkpoint inhibitor can be directed at targets on immune cells but also on tumor cells. Molecular imaging with the ${ }^{89} \mathrm{Zr}$-labeled programmed death ligand 1 checkpoint inhibitor atezolizumab in metastatic triple-negative breast cancer, NSCLC, and urothelial carcinoma showed heterogeneous ${ }^{89} \mathrm{Zr}$-atezolizumab tumor uptake and, interestingly, uptake in lymphoid tissues (38).

mAbs can be modified to serve a specific mechanism of actionfor example, bispecific antibodies directed against a tumor surface antigen and cluster of differentiation $3 \varepsilon$ on T cells. These drugs can be a full-sized $\mathrm{mAb}$ or a modified antibody such as 2 linked, singlechain variable fragments resulting in a $55-\mathrm{kDa}$ bispecific T-cellengaging antibody construct. The results of the biodistribution study with the radiolabeled bispecific T-cell engager ${ }^{89} \mathrm{Zr}$-AMG211, directed against carcinoembryonic antigen in patients with gastrointestinal adenocarcinomas, are awaited (NCT02760199).

\section{ADCs}

ADCs combine high target-specificity with the cytotoxic potential of a chemotherapeutic drug. Currently, 2 ADCs are approved for standard care and more than 50 are in clinical development. In one study, the efficacy of an ADC-targeting carcinoembryonic antigenrelated cell adhesion molecule, CEACAM6, and biodistribution of the naked ${ }^{64} \mathrm{Cu}$-anti-CEACAM6 mAb were assessed in mice with human xenograft pancreatic adenocarcinoma (39). Furthermore, in nonhuman primates, the in vivo distribution showed the highest tracer uptake to be in bone marrow. During treatment with the ADC, all nonhuman primates experienced anemia and thrombocytopenia, suggesting that PET imaging with this mAb predicted the toxicity of its ADC.

There is one clinical imaging study in relation to ADCs. In patients with HER2-positive metastatic breast cancer, a study was performed to assess ${ }^{89} \mathrm{Zr}$-trastuzumab as a biomarker to identify nonresponders to treatment with the ADC trastuzumab emtansine (40). In $29 \%$ of the patients, no ${ }^{89} \mathrm{Zr}$-trastuzumab uptake in tumor lesions was seen. These patients experienced a shorter time to treatment failure than did those with uptake in tumor lesions. The combination of a negative pretreatment ${ }^{89} \mathrm{Zr}$-trastuzumab PET result and absence of response on early ${ }^{18} \mathrm{~F}$-FDG PET performed in the week preceding cycle 2 resulted in a negative predictive value of $100 \%$ for treatment response according to RECIST 1.1 and therefore could potentially be a powerful tool in predicting which patients will not benefit from trastuzumab emtansine treatment. Also, intrapatient heterogeneity, defined as tracer uptake not in all lesions but in a dominant part or minor part of the total tumor load, was detected in $46 \%$ of the patients, providing insight on the extent of this phenomenon.

\section{Blood-Brain Barrier}

Of special interest regarding biodistribution is penetration of the drug across the blood-brain barrier into the CNS. A point of discussion is whether mAbs reach brain metastases to the same extent as they reach extracranial metastases, since mAbs, being of heavy weight, cannot pass the blood-brain barrier. A study with ${ }^{89} \mathrm{Zr}$-bevacizumab and gadolinium-enhanced MRI in 7 children with radiated diffuse intrinsic pontine glioma found heterogeneity in tumor tracer uptake (41). Two tumors showed no tracer uptake. In 4 of 5 tumors, tracer uptake corresponding to contrast-enhanced areas on MRI was seen, as is highly suggestive of leakage in the blood-brain barrier. In another study, with trastuzumab and 
lumretuzumab, specific tracer uptake in multiple brain metastases was seen $(31,34)$. Although clinical evidence is scarce, first results demonstrate the potential of molecular imaging for studying CNS penetration of mAbs.

\section{ANTIHORMONAL THERAPY AND ${ }^{18}$ F-FES PET OR 18F-FDHT PET}

Antihormonal therapy is commonly used in patients with breast and prostate cancer. Although numerous antihormonal treatments are available, there is a constant search for new drugs. In breast cancer, approximately $75 \%$ of the tumors express the estrogen receptor (ER), and patients with such tumors can potentially be treated with antihormonal therapies (42). Tumor ER expression is measured immunohistochemically before such therapy is started. However, heterogeneity can occur between the primary and metastatic sites and between metastases (43). In that case, a single biopsy may not be representative of the ER expression in all lesions. PETmeasured $16 \alpha-{ }^{18} \mathrm{~F}$-fluoro- $17 \beta$-estradiol $\left({ }^{18} \mathrm{~F}-\mathrm{FES}\right)$ tumor uptake correlated well with ER expression by the tumor (44). To get whole-body information on ER expression, radiolabeled estradiol uptake as measured with ${ }^{18} \mathrm{~F}$-FES PET is of interest.

Paradoxically, besides the better-known antiestrogen therapy for ER-positive breast cancer, estrogens can also induce tumor regression. It is thought that long-term estrogen deprivation triggers hypersensitivity to estrogens, with an increase in ER expression. To find patients eligible for estrogen therapy, knowledge about ER expression might be helpful. To this purpose, 19 patients with hormone-resistant metastatic breast cancer underwent baseline ${ }^{18}$ F-FES PET followed by treatment with $2 \mathrm{mg}$ of estradiol 3 times daily, with response assessment in 15 patients (45). An $\mathrm{SUV}_{\max }$ threshold of more than 1.5 was considered as positive uptake (46). Baseline ${ }^{18} \mathrm{~F}$-FES PET uptake produced a positive predictive value of $60 \%$ and a negative predictive value of $80 \%$ for treatment response, suggesting that the ${ }^{18}$ F-FES PET especially identified patients unlikely to benefit from estradiol therapy-those with low or no ${ }^{18} \mathrm{~F}$-FES tumor uptake.

Apart from insight on whole-body uptake of estradiol by tumor lesions, ${ }^{18} \mathrm{~F}$-FES PET is also used during drug development as a pharmacodynamic marker. This was first evaluated for fulvestrant, a pure ER antagonist and a selective ER degrader. Regretfully, more than $50 \%$ of patients with metastatic breast cancer do not benefit from fulvestrant, which might be due in part to administration of an insufficient dose (47). ${ }^{18} \mathrm{~F}$-FES PET was therefore used to study whether the current dose of fulvestrant therapy is sufficient for optimally abolishing estradiol uptake in the tumor (48). Sixteen patients underwent ${ }^{18}$ F-FES PET at baseline and after $4 \mathrm{wk}$ of treatment consisting of $500 \mathrm{mg}$ of fulvestrant on days 1,14 , and 28 . In 6 patients, the predefined relevant $75 \%$ reduction in ${ }^{18} \mathrm{~F}$-FES was not reached. The $38 \%$ of the patients with incomplete reduction of ${ }^{18} \mathrm{~F}$-FES uptake were more likely to develop progressive disease within 24 wk of therapy.

Others have taken a similar approach for a pharmacodynamic readout. $Z$-4-hydroxy- $N$-demethyl-tamoxifen hydrochloride (Z-endoxifen), a selective ER modulator, has been tested in a phase 1 study (49). $Z$-endoxifen is the most potent tamoxifen metabolite and therefore might be more effective than tamoxifen. Patients received $40-300 \mathrm{mg} / \mathrm{d}$ orally for $28 \mathrm{~d}$ per cycle. In 8 of 15 patients who underwent imaging with ${ }^{18} \mathrm{~F}$-FES PET at baseline, uptake in tumor lesions $(n=41)$ was seen $(50)$. In these 8 patients, ${ }^{18} \mathrm{~F}$-FES PET was repeated early after administration of
Z-endoxifen (1-5 d). At a per-patient level, the average $\mathrm{SUV}_{\max }$ among all lesions at baseline (4.8) decreased by $33.6 \%$ after $1-5 \mathrm{~d}$.

GDC-0810 is a novel ER antagonist that binds to the ER and induces conformational changes that lead to receptor degradation (51). ${ }^{18} \mathrm{~F}$-FES PET was used in a phase 1 study to evaluate ER occupancy and guide dose selection (52). Thirty postmenopausal women underwent a baseline scan. The average SUV corrected for background (defined as $\mathrm{SUV}_{\max }-\mathrm{SUV}_{\text {background, derived from }}$ surrounding normal tissue) of lesions per patient at baseline ranged from 0.2 to 9.3 , with a median of 3.1. GDC-0810 was given in different dosages of $200-800 \mathrm{mg}$ daily orally. Followup scanning, performed on 24 patients 4 wk after treatment, demonstrated an impressive reduction in ${ }^{18} \mathrm{~F}$-FES uptake by more than $90 \%$, ranging from $63.6 \%$ to $100 \%$ at the different doses, with a greater ${ }^{18} \mathrm{~F}$-FES reduction at higher-dose groups. Selection of the 600-mg daily dose for phase 2 studies was based on the decrease in ${ }^{18} \mathrm{~F}$-FES uptake, safety, and pharmacokinetics. Further development has been discontinued.

Elacestrant (RAD1901) is a novel selective ER degrader that binds and targets ER for degradation in a dose-dependent manner. ${ }^{18} \mathrm{~F}-\mathrm{FES}$ PET was performed on healthy volunteers at baseline and after $6 \mathrm{~d}$ of RAD1901 treatment at doses of up to $1,000 \mathrm{mg} / \mathrm{d}$ to assess ER engagement. With dosing at 200 and $500 \mathrm{mg} / \mathrm{d}$, a complete attenuation of ${ }^{18} \mathrm{~F}$-FES PET signal was observed in tissues with baseline high uptake, such as the uterus (53). Thereafter, ${ }^{18} \mathrm{~F}-\mathrm{FES}$ PET imaging as an early indicator of clinical response to RAD1901 treatment in breast cancer patients was explored (54). RAD1901 reduced ${ }^{18} \mathrm{~F}$ FES uptake by $79 \%-91 \%$ on day 14 , compared with baseline, in patients given $400 \mathrm{mg} / \mathrm{d}$ (54). For all these studies, it is important to realize that reduction in radiolabeled-estradiol uptake does not necessarily mean that this results in antitumor efficacy.

Another example of molecular imaging for pharmacodynamic assessment during hormonal therapy is ${ }^{18} \mathrm{~F}-16 \beta$-fluoro- $5 \alpha$ dihydrotestosterone $\left({ }^{18} \mathrm{~F}\right.$-FDHT) PET in prostate cancer. ${ }^{18} \mathrm{~F}$-FDHT tumor uptake measured with PET has shown a good correlation with tumor androgen receptor (AR) expression (55). Enzalutamide, an AR antagonist, was selected for clinical development because of several characteristics. AR antagonism was assessed in vitro in a binding assay in which ${ }^{18} \mathrm{~F}-\mathrm{FDHT}$ and the compound of interest competed for AR binding (56). Enzalutamide and another anti-AR compound, RD162, showed 5- to 8-fold greater affinity than bicalutamide in this competition assay. Enzalutamide also showed activity in prostate cancer models with overexpressed AR and bicalutamide resistance (56). In 140 patients, a 30-600 mg/d dosage of enzalutamide was administrated orally (57). ${ }^{18} \mathrm{~F}$-FDHT PET on 22 patients showed less tumor uptake at dosages of $60 \mathrm{mg} / \mathrm{d}$ and above, with an apparent maximal effect seen at $150 \mathrm{mg} / \mathrm{d}$. The phase 3 AFFIRM study, with $160 \mathrm{mg}$ of enzalutamide per day, showed improved overall survival compared with placebo in castration-resistant prostate cancer after chemotherapy (58), and enzalutamide is currently an approved drug for prostate cancer treatment.

\section{OPTICAL IMAGING IN DRUG DEVELOPMENT}

Optical imaging also has a real potential to support drug development. Because of high sensitivity with submillimeter resolution, fluorescent tracers can be studied at a microscopic level. However, given the low penetration depth of whole-body imaging, it is not feasible, and only accessible lesions can be studied. Optical imaging has already been performed with fluorescently labeled mAbs such as bevacizumab in the intraoperative setting (59). Although the 
primary aim was to detect tumor lesions intraoperatively, use of the ex vivo specimens also allowed the study of intratumoral drug distribution. The intratumoral drug distribution showed specific tumor uptake. These approaches stress the potential role of optical imaging in drug development.

\section{CONCLUSION AND FUTURE DIRECTIONS}

With the arrival of novel treatment strategies for cancer, cancer drug development is rapidly expanding and requires enormous resources. Only a small number of the drugs under development obtain approval, with enormous financial costs for those that fail to be approved. Molecular imaging studies provide in vivo insight on drug target expression, pharmacokinetics, and pharmacodynamics. Although these studies are expensive and require time and expertise, valuable information on drug development can be extracted. However, molecular imaging should be complemented by other techniques when relevant, such as pharmacokinetic analysis, as well as radiomics (analysis extracted from PET, CT, or MR images) or analysis of tumor biopsies, circulating tumor DNA, or circulating tumor cells by genomics, transcriptomics, or proteomics. This toolbox of techniques has gained interest in cancer drug development and allows biomarker exploration, patient selection, and insight on mechanism of action.

\section{DISCLOSURE}

Elisabeth G.E. de Vries has a consultation/advisory role with Pfizer. Mathilde Jalving has an advisory role with Merck. Research funding was provided by Amgen, Abbott, Genentech/ Roche, Chugai, Servier, Novartis, Synthon, AstraZeneca, Merck, Pfizer, SMS-Oncology, and Radius Health, all made available to the institution. Elisabeth G.E. de Vries received grant RUG 201610034 from the Dutch Cancer Society and the ERC advanced grant OnQview. No other potential conflict of interest relevant to this article was reported.

\section{REFERENCES}

1. The biopharmaceutical pipeline: innovative therapies in clinical development. Pharmaceutical Research and Manufacturers of America website. http://phrmadocs.phrma.org/files/dmfile/Biopharmaceutical-Pipeline-Full-Report.pdf. Updated July 2017. Accessed February 2, 2018.

2. List of 2015 medicines in development for cancer. Pharmaceutical Research and Manufacturers of America website. http://phrma-docs.phrma.org/sites/default/files/ pdf/2015_cancer_drug_list.pdf. Updated August 21, 2015. Accessed February 2, 2018.

3. List of 2017 immuno-oncology medicines in development. Pharmaceutical Research and Manufactures of America website. http://phrma-docs.phrma.org/files/ dmfile/MID_Immuno-Oncology-2017_Drug-List1.pdf. Updated May 26, 2017. Accessed February 2, 2018.

4. Brawley L. With 20 agents, 803 trials, and 166,736 patient slots, is pharma investing too heavily in PD-1 drug development? Cancer Lett. 2016;42:2-18.

5. DiMasi JA, Grabowski HG, Hansen RW. Innovation in the pharmaceutical industry: new estimates of R\&D costs. J Health Econ. 2016;47:20-33.

6. Prasad V, Mailankody S. Research and development spending to bring a single cancer drug to market and revenues after approval. JAMA Intern Med. 2017;177: 1569-1575.

7. Morgan P, van der Graaf PH, Arrowsmith J, et al. Can the flow of medicines be improved? Fundamental pharmacokinetic and pharmacological principles toward improving phase II survival. Drug Discov Today. 2012;17:419-424.

8. Banerji U, Workman P. Critical parameters in targeted drug development: the pharmacological audit trail. Semin Oncol. 2016;43:436-445.

9. Burt T, Yoshida K, Lappin G, et al. Microdosing and other phase 0 clinical trials: facilitating translation in drug development. Clin Transl Sci. 2016;9:74-88.

10. Slobbe P, Poot AJ, Windhorst AD, van Dongen GA. PET imaging with smallmolecule tyrosine kinase inhibitors: TKI-PET. Drug Discov Today. 2012;17:1175-1187.
11. Brastianos PK, Carter SL, Santagata S, et al. Genomic characterization of brain metastases reveals branched evolution and potential therapeutic targets. Cancer Discov. 2015;5:1164-1177.

12. Song Y, Xiao Z, Wang K, et al. Development and evaluation of ${ }^{18} \mathrm{~F}$-IRS for molecular imaging mutant EGF receptors in NSCLC. Sci Rep. 2017;7:3121.

13. Genne P, Berthet C, Raguin O, et al. Preclinical proof of concept for the first nanocyclix TKI-PET radiotracer targeting activated EGFR positive lung tumors [abstract]. Cancer Res. 2017;77(13, suppl):1875A.

14. Bahce I, Smit EF, Lubberink M, et al. Development of $\left[{ }^{11} \mathrm{C}\right]$ erlotinib positron emission tomography for in vivo evaluation of EGF receptor mutational status. Clin Cancer Res. 2013;19:183-193.

15. Bahce I, Yaqub M, Errami H, et al. Effects of erlotinib therapy on $\left[{ }^{11} \mathrm{C}\right]$ erlotinib uptake in EGFR mutated, advanced NSCLC. EJNMMI Res. 2016;6:10.

16. Memon AA, Weber B, Winterdahl M, et al. PET imaging of patients with nonsmall cell lung cancer employing an EGF receptor targeting drug as tracer. $\mathrm{Br} \mathrm{J}$ Cancer. 2011;105:1850-1855.

17. Meng X, Loo BW Jr, Ma L, Murphy JD, Sun X, Yu J. Molecular imaging with ${ }^{11} \mathrm{C}$-PD153035 PET/CT predicts survival in non-small cell lung cancer treated with EGFR-TKI: a pilot study. J Nucl Med. 2011;52:1573-1579.

18. Ballard P, Yates JW, Yang Z, et al. Preclinical comparison of osimertinib with other EGFR-TKIs in EGFR-mutant NSCLC brain metastases models, and early evidence of clinical brain metastases activity. Clin Cancer Res. 2016;22:51305140.

19. Mok TS, Wu YL, Ahn MJ, et al. Osimertinib or platinum-pemetrexed in EGFR T790M-positive lung cancer. N Engl J Med. 2017;376:629-640.

20. Zeng Q, Wang J, Cheng Z, et al. Discovery and evaluation of clinical candidate AZD3759, a potent, oral active, central nervous system-penetrant, epidermal growth factor receptor tyrosine kinase inhibitor. J Med Chem. 2015;58:8200-8215.

21. Ahn M-J, Kim D-W, Cho BC, et al. Phase I study (BLOOM) of AZD3759, a BBB penetrable EGFR inhibitor, in patients with TKI-naïve, EGFRm NSCLC with CNS metastases [abstract]. J Clin Oncol. 2017;35(15, suppl):2006.

22. Soda M, Choi YL, Enomoto M, et al. Identification of the transforming EML4ALK fusion gene in non-small-cell lung cancer. Nature. 2007;448:561-566.

23. Zou HY, Friboulet L, Kodack DP, et al. PF-06463922, an ALK/ROS1 inhibitor, overcomes resistance to first and second generation ALK inhibitors in preclinical models. Cancer Cell. 2015;28:70-81.

24. Gadgeel SM, Gandhi L, Riely GJ, et al. Safety and activity of alectinib against systemic disease and brain metastases in patients with crizotinib-resistant ALKrearranged non-small-cell lung cancer (AF-002JG): results from the dose-finding portion of a phase 1/2 study. Lancet Oncol. 2014;15:1119-1128.

25. Peters S, Camidge DR, Shaw AT, et al. Alectinib versus crizotinib in untreated ALK-positive non-small-cell lung cancer. N Engl J Med. 2017;377:829-838.

26. Johnson TW, Richardson PF, Bailey S, et al. Discovery of (10R)-7-amino-12fluoro-2,10,16-trimethyl-15-oxo-10,15,16,17-tetrahydro-2H-8,4-(metheno)pyrazolo[4,3-h][2,5,11]-benzoxadiazacyclotetradecine-3-carbonitrile (PF-06463922), a macrocyclic inhibitor of anaplastic lymphoma kinase (ALK) and c-ros oncogene 1 (ROS1) with preclinical brain exposure and broad-spectrum potency against ALK-resistant mutations. J Med Chem. 2014;57:4720-4744.

27. Collier TL, Normandin MD, Stephenson NA, et al. Synthesis and preliminary PET imaging of ${ }^{11} \mathrm{C}$ and ${ }^{18} \mathrm{~F}$ isotopologues of the ROS1/ALK inhibitor lorlatinib. Nat Commun. 2017;8:15761.

28. Carney B, Kossatz S, Reiner T. Molecular imaging of PARP. J Nucl Med. 2017;58:1025-1030.

29. Edmonds CE, Makvandi M, Lieberman BP, et al. $\left({ }^{18} \mathrm{~F}\right)$ fluorthanatrace uptake as a marker of PARP1 expression and activity in breast cancer. Am J Nucl Med Mol Imaging. 2016;6:94-101.

30. Makvandi M, Pantel A, Schwartz LE, et al. Exploring the significance of PARP-1 expression for therapy and clinical PET/CT imaging of PARP-1 in ovarian cancer [abstract]. Cancer Res. 2017;77(13, suppl):3716.

31. Dijkers EC, Oude Munnink TH, Kosterink JG, et al. Biodistribution of ${ }^{89} \mathrm{Zr}$ trastuzumab and PET imaging of HER2-positive lesions in patients with metastatic breast cancer. Clin Pharmacol Ther. 2010;87:586-592.

32. Bruno R, Washington CB, Lu JF, Lieberman G, Banken L, Klein P. Population pharmacokinetics of trastuzumab in patients with HER2 + metastatic breast cancer. Cancer Chemother Pharmacol. 2005;56:361-369.

33. Gaykema SB, de Jong JR, Perik PJ, et al. ${ }^{111}$ In-trastuzumab scintigraphy in HER2-positive metastatic breast cancer patients remains feasible during trastuzumab treatment. Mol Imaging. 2014;13:1-6.

34. Bensch F, Lamberts LE, Smeenk MM, et al. ${ }^{89} \mathrm{Zr}$-lumretuzumab PET imaging before and during HER3 antibody lumretuzumab treatment in patients with solid tumors. Clin Cancer Res. 2017;23:6128-6137.

35. Bahce I, Huisman MC, Verwer EE, et al. Pilot study of ${ }^{89} \mathrm{Zr}$-bevacizumab positron emission tomography in patients with advanced non-small cell lung cancer. EJNMMI Res. 2014;4:35 
36. Oosting SF, Brouwers AH, van Es SC, et al. ${ }^{89} \mathrm{Zr}$-bevacizumab PET visualizes heterogeneous tracer accumulation in tumor lesions of renal cell carcinoma patients and differential effects of antiangiogenic treatment. J Nucl Med. 2015;56:63-69.

37. van Asselt SJ, Oosting SF, Brouwers $\mathrm{AH}$, et al. Everolimus reduces ${ }^{89} \mathrm{Zr}$ bevacizumab tumor uptake in patients with neuroendocrine tumors. $\mathrm{J} \mathrm{Nucl}$ Med. 2014;55:1087-1092.

38. Bensch F, van der Veen E, Jorritsma A, et al. First-in-human PET imaging with the PD-L1 antibody ${ }^{89} \mathrm{Zr}$-atezolizumab [abstract]. Cancer Res. 2017;77(13, suppl): CT017.

39. Strickland LA, Ross J, Williams S, et al. Preclinical evaluation of carcinoembryonic cell adhesion molecule (CEACAM) 6 as potential therapy target for pancreatic adenocarcinoma. J Pathol. 2009;218:380-390.

40. Gebhart G, Lamberts LE, Wimana Z, et al. Molecular imaging as a tool to investigate heterogeneity of advanced HER2-positive breast cancer and to predict patient outcome under trastuzumab emtansine (T-DM1): the ZEPHIR trial. Ann Oncol. 2016;27:619-624.

41. Jansen MH, Veldhuijzen van Zanten SEM, van Vuurden DG, et al. Molecular drug imaging: ${ }^{89} \mathrm{Zr}$-bevacizumab PET in children with diffuse intrinsic pontine glioma. J Nucl Med. 2017;58:711-716.

42. Blamey RW, Hornmark-Stenstam B, Ball G, et al. ONCOPOOL: a European database for 16,944 cases of breast cancer. Eur J Cancer. 2010;46:56-71.

43. Hoefnagel LD, van der Groep P, van de Vijver MJ, et al. Discordance in ER $\alpha, P R$ and HER2 receptor status across different distant breast cancer metastases within the same patient. Ann Oncol. 2013;24:3017-3023.

44. Peterson LM, Mankoff DA, Lawton T, et al. Quantitative imaging of estrogen receptor expression in breast cancer with PET and ${ }^{18} \mathrm{~F}$-fluoroestradiol. $\mathrm{J} \mathrm{Nucl}$ Med. 2008;49:367-374.

45. van Kruchten M, Glaudemans AW, de Vries EF, Schroder CP, de Vries EG, Hospers GA. Positron emission tomography of tumour $\left[{ }^{18} \mathrm{~F}\right]$ fluoroestradiol uptake in patients with acquired hormone-resistant metastatic breast cancer prior to oestradiol therapy. Eur J Nucl Med Mol Imaging. 2015;42:1674-1681.

46. van Kruchten M, de Vries EG, Brown M, et al. PET imaging of oestrogen receptors in patients with breast cancer. Lancet Oncol. 2013;14:e465-e475.

47. Di Leo A, Jerusalem G, Petruzelka L, et al. Results of the CONFIRM phase III trial comparing fulvestrant $250 \mathrm{mg}$ with fulvestrant $500 \mathrm{mg}$ in postmenopausal women with estrogen receptor-positive advanced breast cancer. J Clin Oncol. 2010;28:4594-4600.

48. van Kruchten M, de Vries EG, Glaudemans AW, et al. Measuring residual estrogen receptor availability during fulvestrant therapy in patients with metastatic breast cancer. Cancer Discov. 2015;5:72-81.
49. Goetz MP, Suman VJ, Reid JM, et al. First-in-human phase I study of the tamoxifen metabolite Z-endoxifen in women with endocrine-refractory metastatic breast cancer. J Clin Oncol. 2017;35:3391-3400.

50. Lin FI, Gonzalez EM, Kummar S, et al. Utility of ${ }^{18} \mathrm{~F}$-fluoroestradiol ( ${ }^{18} \mathrm{~F}-\mathrm{FES}$ ) $\mathrm{PET} / \mathrm{CT}$ imaging as a pharmacodynamic marker in patients with refractory estrogen receptor-positive solid tumors receiving Z-endoxifen therapy. Eur J Nucl Med Mol Imaging. 2017;44:500-508.

51. Lai A, Kahraman M, Govek S, et al. Identification of GDC-0810 (ARN-810), an orally bioavailable selective estrogen receptor degrader (SERD) that demonstrates robust activity in tamoxifen-resistant breast cancer xenografts. $J$ Med Chem. 2015;58:4888-4904.

52. Wang Y, Ayres KL, Goldman DA, et al. ${ }^{18} \mathrm{~F}$-fluoroestradiol PET/CT measurement of estrogen receptor suppression during a phase I trial of the novel estrogen receptor-targeted therapeutic GDC-0810: using an imaging biomarker to guide drug dosage in subsequent trials. Clin Cancer Res. 2017;23:3053-3060.

53. Harb WA, Garner F, Clarkin M, et al. A phase I study of RAD1901, an oral selective estrogen receptor degrader, in ER-positive, HER2-negative, postmenopausal advanced breast cancer patients [abstract]. J Clin Oncol. 2015;33(15, suppl):TPS638.

54. de Vries EGE, Venema CM, Glaudemans AWJM, et al. A phase 1 study of RAD1901, an oral selective estrogen receptor degrader, to determine changes in the ${ }^{18} \mathrm{~F}$-FES uptake and tumor responses in ER-positive, HER2-negative, advanced breast cancer patients [abstract]. Cancer Res. 2017;77(15 suppl): P2-08-08.

55. Beattie BJ, Smith-Jones PM, Jhanwar YS, et al. Pharmacokinetic assessment of the uptake of $16 \beta-{ }^{18} \mathrm{~F}$-fluoro-5 $\alpha$-dihydrotestosterone (FDHT) in prostate tumors as measured by PET. J Nucl Med. 2010;51:183-192.

56. Tran C, Ouk S, Clegg NJ, et al. Development of a second-generation antiandrogen for treatment of advanced prostate cancer. Science. 2009;324:787-790.

57. Scher HI, Beer TM, Higano CS, et al. Antitumour activity of MDV3100 in castration-resistant prostate cancer: a phase 1-2 study. Lancet. 2010;375:14371446.

58. Scher HI, Fizazi K, Saad F, et al. Increased survival with enzalutamide in prostate cancer after chemotherapy. N Engl J Med. 2012;367:1187-1197.

59. Lamberts LE, Koch M, de Jong JS, et al. Tumor-specific uptake of fluorescent bevacizumab-IRDye800CW microdosing in patients with primary breast cancer: a phase I feasibility study. Clin Cancer Res. 2017;23:2730-2741.

60. van Es SC, Brouwers AH, Mahesh SVK, et al. ${ }^{89} \mathrm{Zr}$-bevacizumab PET: potential early indicator of everolimus efficacy in patients with metastatic renal cell carcinoma. J Nucl Med. 2017;58:905-910. 